\title{
Texture Anisotropy, Symmetry, Regularity: Recovering Structure and Orientation from Interaction Maps ${ }^{1}$
}

\author{
Dmitry Chetverikov \\ MTA SzTAKI, Budapest, P.O.Box 63, H-1518 Hungary \\ Robert M. Haralick \\ University of Washington, Seattle, WA 98195, USA
}

\begin{abstract}
We discuss a novel method for recovering fundamental, perceptually motivated structural features of a texture pattern: anisotropy, symmetry, and regularity. The method is based on extended spatial grey-level difference statistics which describe pairwise pixel interactions and yield an interaction map used to assess the overall two-dimensional structure of interactions and extract the significant short- and long-range interactions (intersample spacings). The new approach extends, in digital images, the notion of greylevel difference to arbitrary spacing vectors (i.e. any angle at any displacement). This provides the necessary background for precise anisotropy (or directionality) and symmetry analysis. Experimental results are shown with a set of Brodatz images that range from highly regular to patterns with weak regularity or anisotropy. A few especially interesting examples of recovering hardly visible structural features are given. Finally, the approach is applied to rotation-invariant texture classification.
\end{abstract}

\section{Introduction}

There is strong psychophysical evidence that the attentive texture perception by humans relies on such fundamental properties as anisotropy (directionality), symmetry, regularity and structural complexity. A recent paper by Rao and Lohse [1] reports on a study of texture perception aimed at identifying those high level texture features that account for most of the attentive texture discrimination capability of the human vision. It is concluded that directionality and regularity are among those very few high level texture features that guide the process of perceptual grouping (taxonomy) of textural patterns. In spite of the recent doubts in the preattentive-attentive dichotomy (e.g. [2]), the perceptional value of the fundamental texture features is not questioned.

Let us briefly discuss the current trends in the computer analysis of the fundamental structural properties of textures grouping the papers around the high-level features identified in [1]. More information on early approaches to structure can be found in [3] and [4].

${ }^{1}$ This work was supported in part by the grant OTKA T14520. 
Recently, there has been growing interest in the so-called oriented patterns such as texture images originating from flow-like processes. Directionality has become a popular topic of texture research. (See e.g. [5-11].) Practical motivations for directionality assessment include, among others, flow research [7], image database query [9] and orientation invariant texture classification [10]. Computer analysis of this textural property usually involves (multiscale) orientation-sensitive filtering followed by coherence evaluation of local orientation [5-10]. The analysis reveals the directionality that stems from the dominating orientation of elongated texture elements. This is a perceptually important component of anisotropy. In terms of texture elements, however, both anisotropy and regularity may have at least three components: that of the placement rules, that of the shape/orientation, and that of the intensity distribution of texels. These components may interact producing more complex anisotropic patterns, while most of the above approaches are aimed at detecting a single perceptually dominant direction assuming an unidirectional pattern. To our best knowledge, anisotropy in general was investigated in just a few early works including [12] and [13] where the notion of anisotropy indicatrix was introduced and applied to rotation invariant texture classification.

The relevance of anisotropy is mainly due to its relation to symmetry with its universal role in nature in general and human and computer vision in particular. (See [14] for an excellent collection of related papers.) Basic conservation laws of physics follow from symmetry properties of space-time. Analysis of a physical process can often be reduced to separable components if a proper co-ordinate system is set that complies with the spatial symmetry and anisotropy of the process. Obviously, this also applies to the processes that create natural and artificial textures, hence to simplify their analysis one has to find the axes of texture anisotropy.

The increasing number of studies on symmetry of shapes and local intensity patterns reflect the understanding of the importance of symmetry for computer vision. Recently, symmetry has been applied to textures as well. Most of the related algorithms (e.g. $[2,15])$ use a symmetry operator to detect local intensity symmetries in textures. An example of perceptually motivated detection of global reflectional symmetry in texturelike patterns can be found in [16]. In this study coherent groups of oriented local elements are matched across a hypothesised axis of symmetry. A different, statistical view of texture symmetry is advocated in our recent paper [17]: global texture symmetry is revealed as the symmetry of a polar diagram called grey level feature based anisotropy indicatrix. In the present study we will use the approach [17] as the starting point in order to define a heuristic interaction map.

The necessity to analyse a pattern at both short and long ranges in a variety of directions has been realised in early papers on statistical approaches to texture regularity [18]. However in practice, due to the limitations of the co-occurrence matrix, the studies

were confined to the multiples of $\pi / 4$ and pre-oriented patterns (e.g. [19]). Often, small neighbourhoods were only used which excludes the possibility of discovering long range regularity. In more recent attempts (e.g. [20]), finer angular search is combined with longer distances that allow for the finer angular resolution. However, small distances that can be important for anisotropy analysis are practically lost. This is related to another typical drawback of the algorithms, namely their incapability to detect weak structure and provide transition from regularity to anisotropy. Anisotropy is a lower 
level description of structure. Under gradual degradation of a pattern, one would expect a structural analyser to give a continuous indication of going from the higher to the lower level [12,21].

Among approaches to structure, a distinct group is formed by the so-called structural methods that view texture as a spatial arrangement of small elementary regions, or primitives. Since the methods proposed in this paper are pixel-based, we will discuss neither region-based nor local intensity feature (e.g. edge) based structural methods. The dramatic decrease since the mid-eighties of work on region-based structural techniques illustrates the fact that many textural patterns cannot be represented by elements. Even when the elements are perceived by humans, they are hard to extract by computer without prior knowledge of their properties. The reader is referred to [4] for a related survey and a description of one of a few implemented and tested structural systems.

The main message of this paper is that the pairwise pixel interactions carry very important structural information and, by investigating the spatial dependence of the cooccurrence or grey-level difference features, this information can be accessed more efficiently than by trying to select the "best" features for a limited number of pre-set spacings. We present and experimentally study a heuristic method for recovering such fundamental structural properties of textures as anisotropy, symmetry and regularity. The approach uses the notion of the spatial grey-level difference based anisotropy indicatrix [17] which is useful for precise anisotropy and symmetry analysis. A sequence of indicatrices yields an interaction map which reflects the overall structure of pixel interactions. The map facilitates the extraction of the characteristic spacing vectors. The experimental analysis shows that it can be used to recover the pattern structure, including weak regularity and anisotropy. The discriminative power of the map is demonstrated by its efficiency in rotation-invariant texture classification.

Section 1 is devoted to the definition of the model based interaction map. More technical details can be found in previous papers. In section 2 , we demonstrate a variety of characteristic examples of interaction maps and pattern orientation results computed for a collection of the Brodatz images ranging from highly regular, multidirectional textures to less regular unidirectional patterns. The results of the rotation-invariant texture classification are shown in section 3. Finally, the advantages and the limits of the proposed method are discussed in section 4 .

\section{The Interaction Map}

Motivated by the discovery of the perceptual value of anisotropy and the growing number of related computational approaches to pattern directionality we have recently extended the notion of co-occurrence to arbitrary spacing [17]. The objective was to make the magnitude of the spacing vector (the displacement) independent of the vector angle thus providing the possibility of detailed anisotropy analysis at both short and long ranges. The idea of the extended grey level difference histogram (EGLDH) introduced in [17] is straightforward. If the spacing vector points at a non-integer location, one can estimate the grayvalue in this location by interpolating the values of the neighbouring pixels. In the image raster, we do this for each discrete position of the origin of the spacing vector. The difference of the two grayvalues connected by the 
spacing vector is used to increment the corresponding entry of the EGLDH. The same interpolation procedure can be used to extend the co-occurrence probability matrix as well. See [17] for details.

Texture images may have both short- and long-range pixel interactions of significant strength. The interaction map shows "at a glance" the significance of any particular spacing. The relevance of different pairwise interactions (cliques) can be measured in the framework of the Markov/Gibbs model with multiple pairwise pixel interactions which has been recently introduced by Gimel'farb in [22]. The model-based maps discussed in [22] are related to the EGLDH feature-based interaction maps we are going to introduce now. The model [22] supports our feature-based approach by stating that, under the model assumptions, the spatial grey-level difference histograms considered by both approaches are the sufficient statistics for this model. The main steps and definitions of the EGLDH feature-based interaction map approach are as follows:

Step 1. For a discrete set of spacing vectors $\boldsymbol{d}_{i j}=\left(\alpha_{i}, d_{j}\right), \alpha_{i} \in[0,2 \pi]$, $d_{j} \in\left[1, d_{\max }\right]$, compute an EGDLH feature $F\left(\alpha_{i}, d_{j}\right)$.

Step 2. Define polar interaction map as $M_{p l}(i, j)=F\left(\alpha_{i}, d_{j}\right)$. Each column of $M_{p l}(i, j)$ is called anisotropy indicatrix describing texture anisotropy at the distance $d_{j}: A^{j}(i)=M_{p l}(i, j) \cdot A^{j}(i)$ is treated as a cyclical array, i.e. modulo $i_{\max }$.

Step 3. Apply a reflectional symmetry transform to each indicatrix $A^{j}(i)$, $j=1, \ldots, j_{\max }$, to obtain the corresponding symmetry indicatrix $S^{j}(i)$.

Step 4. Define polar symmetry map as $S_{p l}(i, j)=S^{j}(i)$.(The indicatrices form the columns of the map.)

Step 5. Compute row projections of $S_{p l}(i, j)$ to find directions of dominant symmetry and evaluate overall anisotropy and orientation.

Step 6. Transform $M_{p l}(i, j)$ to XY coordinates to obtain Cartesian interaction map $M_{x y}(m, n)$. Use $M_{x y}(m, n)$ to analyse texture structure.

In step 1, any of the standard GLDH features [3] can be computed for the extended graylevel difference histograms. In this paper, we use the median as the EGLDH feature $F\left(\alpha_{i}, d_{j}\right)$. The median feature is defined as the median of the absolute differences of a pair of gray levels separated by the spacing vector $\boldsymbol{d}_{i j}$. The range of the displacements $d_{j}$ should be wide enough to include the expected 
characteristic values, e.g. the period of a regular pattern. The angular resolution depends on the desired accuracy of anisotropy analysis.

In the initial study [17] we considered a sequence of anisotropy indicatrices

$A^{j}(i)$ obtained for a range of displacements. An indicatrix was depicted as a polar diagrams representing the magnitude of the EGLDH feature in each direction by the radius, i.e. the distance from the origin. An interaction map is depicted as a grayscale image where the magnitude of the feature is coded by intensity. A single map represents a sequence of indicatrices and gives an overall view of structure and strengths of pairwise interactions.

The idea of investigating a GLDH feature as a function of the spacing vector is not new (see e.g. [18],[19]), but it had not been realised properly until the histogram was extended to non-integer spacing vectors. Traditionally, the emphasis was laid on the design or selection of particular features rather than on the mapping a feature onto the spacing vector space. For the current approach, the choice of a particular feature is less important than the mapping which reveals the structure of the pattern.

Given an anisotropy indicatrix, in step 3 we evaluate the reflectional symmetry of the indicatrix with respect to a sequence of axes with the orientation $\alpha_{i}$ that pass through the indicatrix origin. For this purpose, we match the two halves of the indicatrix defined by each axis. The matching rate is used as a measure of reflectional texture symmetry in the direction $\alpha_{i}$. The polar diagram depicting the matching rate is viewed as a symmetry-based anisotropy indicatrix. (See [17] for details and definitions.)

Figure 1 demonstrates the interaction maps obtained for a regular texture pattern from the Brodatz album [24]. The angular resolution is 5 degrees, the maximum displacement 30 pixels. The maps are presented as scaled intensity-coded images. The relevant features of an interaction map are its dominant blobs (darks pots) and lines. The blobs show the characteristic spacings, e.g. the strongest pixel interactions, while the lines exhibit the pattern anisotropy. Pattern anisotropy and orientation are best expressed by the polar symmetry maps as distinct light rows comprised by the maxima of symmetry. An algorithm for computation of the pattern orientation using the polar symmetry map of the pattern is given elsewhere [23]. The algorithm is based on the analysis of the row projections of the map. An important feature of the algorithm is that for structured patterns it yields the orientation of the structure assessed via the directions of maximum symmetry, as opposed to the traditional local filtering based methods which are incapable of grasping the structure.
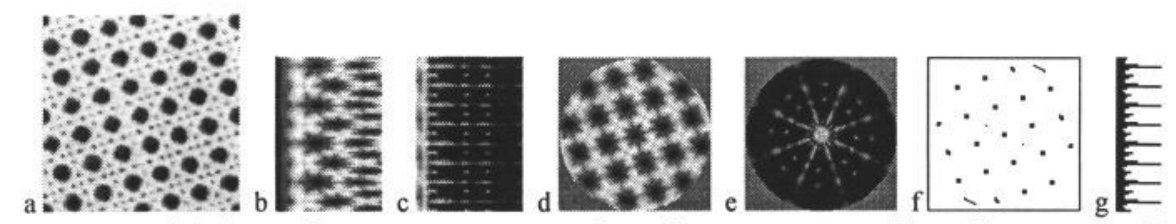

Figure 1. (a) A regular texture pattern; the polar interaction (b) and symmetry (c) maps; the Cartesian interaction (d) and symmetry (e) maps; $(f)$ the blobs of $(d) ;(g)$ the row projections of (c). The polar maps and the projections are enlarged. 


\section{Experimental Analysis of Structure, Symmetry, and Orientation}

In this section we experimentally demonstrate that the indicatrices and the interaction maps can be used to recover the fundamental structural features of texture patterns. When selecting the images we tried to test the power and the limits of the proposed solutions by including weak regularity images taken at random orientations. The interaction maps have been computed for most of the Brodatz images, but only those of the results that we believe are most illustrative are presented here. More examples will be given in a forthcoming journal paper.

Figure 2 illustrates the capabilities of the proposed method in recovering the structure and orientation of texture patterns. Each entry of the figure (except the last one) shows a texture sample with the orientation analysis results overlaid and the blobs of the interaction map displayed separately. Any of the anisotropy (symmetry) axes depicted can be used to orient the pattern. Examples (a) and (b) are directional nonregular textures whose orientation is easily detectable by both traditional filtering techniques and the proposed approach. In this case, the interaction maps themselves are shown because the maps have linear rather than blob structure. Examples (c)-(f) are rectangular structures. Some of them (e.g. (e), see [4]) are likely to pose problems to the existing methods. The handmade paper (d) has structure so weak that is perceived only with effort. To test this result, e.g. to check if it is not an artefact of the method due to the regularity of the raster, we rotated the original photograph in front of the camera and obtained the same (rotated) rectangular structure as shown. When the structure is a square grid, the diagonal axes have the same degree of symmetry as the principal ones. To select the principal axes, further analysis of the structure is needed.

Examples (g)-(i) demonstrate the recovery of hexagonal structures. The last of them (plastic pellets) is hard to perceive. The orientation of the ( $\mathrm{g}$ ) is easier to find because the cells are elongated. The last two examples (j) and (k) show more sophisticated structures. Straw matting (j) exhibits in a non-orthogonal combination (interference) of two anisotropies: that of the geometry (placement rules) and that of the intensity distribution (painting function). Both anisotropies have been detected. The final example $(\mathrm{k})$ is a hierarchical pattern where most local approaches tend to detect only one component of directionality. Our approach is able to detect and separate both, at short and long ranges. (See [23] for details of the method.) In general case, however, the problem of hierarchical textures is still open.
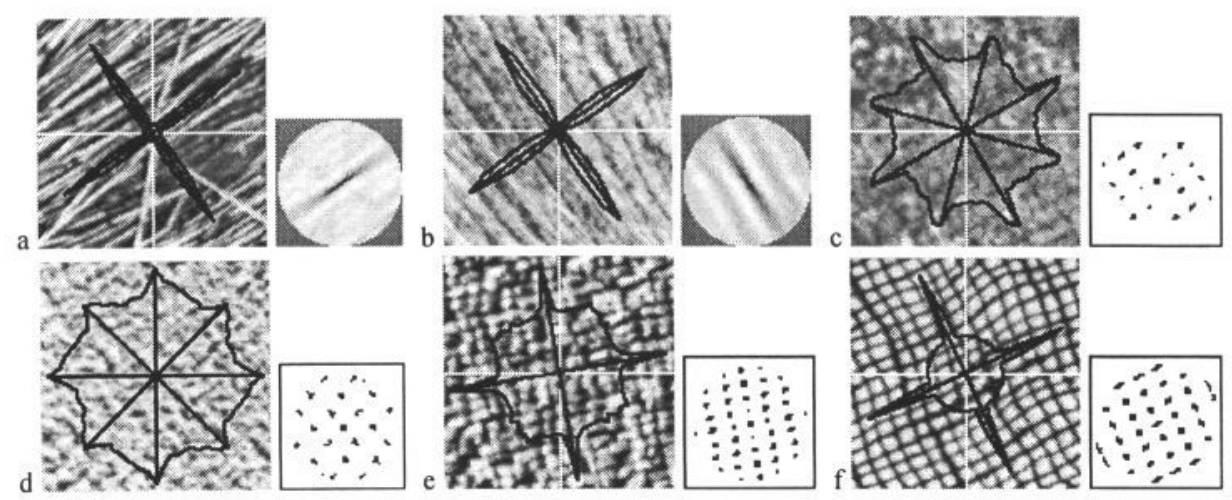

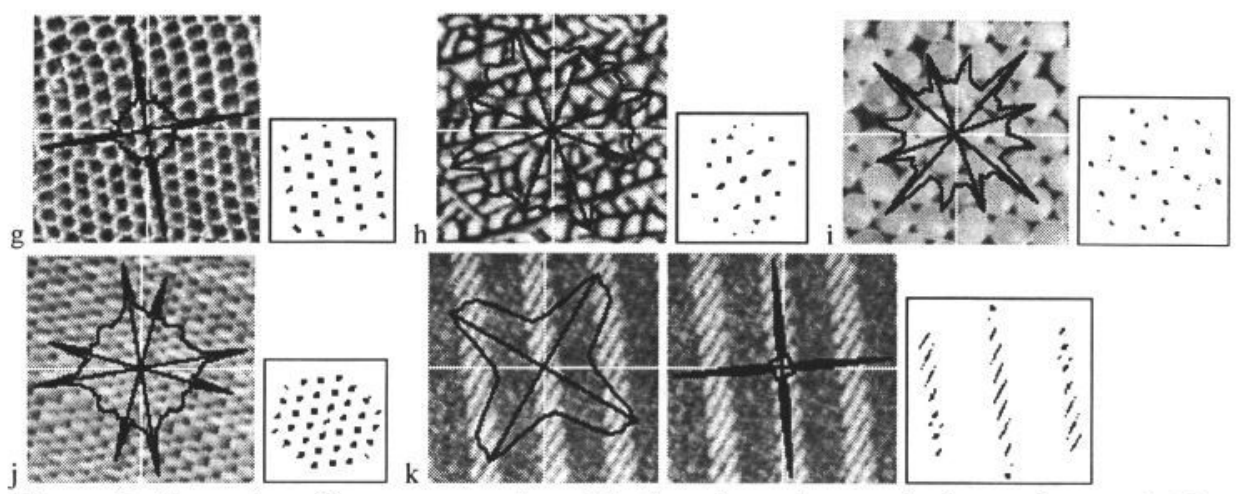

Figure 2. Examples of texture samples with the orientation analysis results overlaid and the blobs of the interaction maps shown separately.

\section{Rotation-Invariant Texture Classification}

To demonstrate the texture discriminating power of the proposed approach, we carried out classification experiments, both with fixed and varying pattern orientations. The experimental protocol was as follows. Thirty textures from the Brodatz album were selected and digitised to $512 \times 512 \times 8$ resolution test images. Each image covered the whole original album photograph. Small samples of the test images are displayed in figure 4. The experimental texture set contained patterns with different degree of regularity as well as non-regular directional patterns. No isotropic, non-regular textures were included since they do not have characteristic interaction maps suitable for discrimination. This is a basic limitation of the proposed approach as far as classification is concerned.

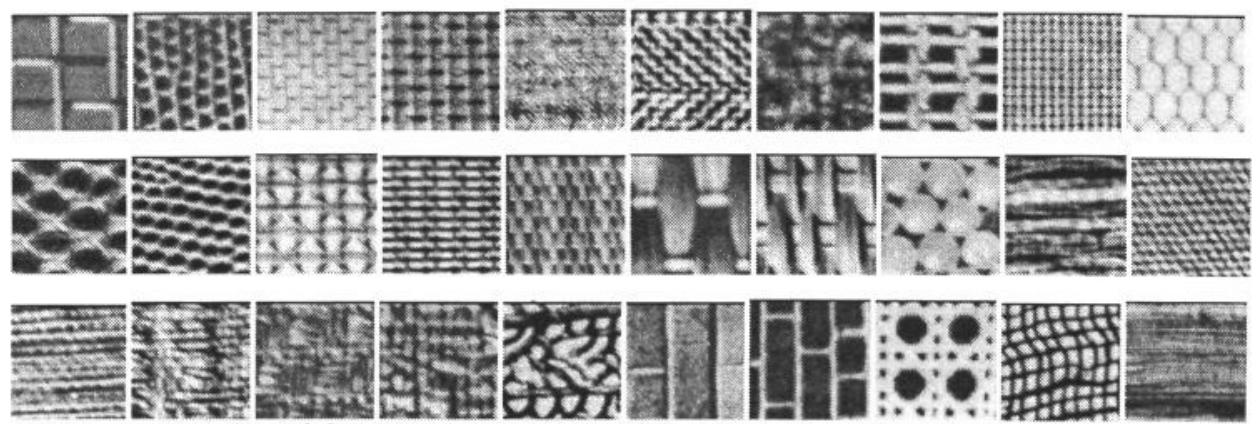

Figure 3. Samples of the texture images used in the classification experiments.

Each of the $M$ test images $(M=30)$ was divided into $K$ non-overlapping subimages ( $K$ was 4 or 9 ). A sample patch was randomly selected from each of the subimages and the polar interaction map of each of the $M K$ samples was computed. For rotation-invariant classification, the orientation of the patch was random. Then the distance between each pair of the $M K$ maps was obtained for a set of relative orientations of the two maps. (In polar maps, rotation means permutation of the rows.) The distance between two maps was defined as the mean absolute gray level difference 
between the corresponding points. The best match, e.g. the smallest distance was selected within a specified set of orientations. In the case of rotation-invariant classification, the relative rotation covered the whole possible range of angles. For fixed orientation, we still applied a limited angular variation ( \pm 30 degrees) in order to compensate for the spatial variations of some textures such as the second one in figure 4 (reptile skin). For the same reasons, slight spatial scaling of the maps would also be desirable in order to achieve more flexible matching, and we plan to add it in future.

In each class, one patch was used as the training sample and the rest $K-1$ ones as the test samples. This was done for each patch of each class and the nearest neighbour was selected in each case. Such classification procedure provides a simple but strict test under unfavourable conditions: the within-class distances should be smaller than the distances to the samples belonging to other classes, i.e. the classes should form distinct clusters. No attempt to apply more sophisticated learning and classification schemes was made, since we wished to test the "descriptive resolution" of interaction maps, not a classification scheme.

The classification test was run 8 times. The results are summarised in Tables 1 and 2 . The classification results were obtained under different conditions: fixed/arbitrary rotation, patch size (110 or 165), image resolution (original or half), spacing vector resolution (original or half). Arbitrary rotation means rotation-invariant classification. The image resolution was reduced using a Gaussian pyramid. The resolution of the polar spacing vector was modified by changing the increments from 5 to 10 degrees for the angle and 1 to 2 pixels for the magnitude. The maximum displacement was 50 or 25 pixels, depending on image resolution. Since we are primarily interested in rotationinvariant classification, the influence of the resolution parameters was tested only in this case.

Table 1. Classification results (\%) for patch size 110 pixels

Rotation-invar. Fixed rotation Rotation-invar. Rotation-invar. original resol. original resol. half image resol. half spacing resol.

$\begin{array}{llll}79 & 82 & 73 & 70\end{array}$

Table 2. Classification results (\%) for patch size 165 pixels $\begin{array}{llll}\begin{array}{l}\text { Rotation-invar. } \\ \text { original resol. }\end{array} & \begin{array}{l}\text { Fixed rotation } \\ \text { original resol. }\end{array} & \begin{array}{l}\text { Rotation-invar. } \\ \text { half image resol. }\end{array} & \text { half spacing resol. }\end{array}$ 96 96 92 87

The classification results for large patches are a few percent better than those presented in a recent study by Greenspan et.al. [25] where the best figure obtained for rotationinvariant discrimination of 30 textures was $92 \%$. However, it is hard to compare the results of the two studies since they were obtained for different texture sets using 
different classification techniques. In [25], the learning/classification scheme is much less strict (12 training and 4 testing samples, more elaborated classification algorithm), but the patch size is smaller (64 pixels). In our case, a relatively large patch size was important because to obtain a meaningful interaction map of a structured pattern the method needs significant pixel statistics at spacings greater than the period of the structure. This is not a deficiency of the proposed approach: long range order can only be detected at long ranges. If the window "sees" less than one period, no method can grasp the periodicity. Alternatively, one may try a multiresolution scheme, but then important image details may be lost at lower resolutions.

The classification results show improvement for larger patch size when the necessary statistics is gained. The growing patch size limits the number of samples, especially in the case of rotation-invariant classification when an arbitrarily rotated sample is selected in a subimage. To check the stability of the results, we ran an additional test with fixed orientation, large patch size, and $K=9$. The figure obtained $(92 \%)$ was reasonably close to that obtained for $K=4$.

Most of the classification errors were caused by a small group of non-regular patterns whose maps are not distinct enough. The detailed analysis of the classification results, including errors, is outside the scope of this paper. It will be given in a forthcoming journal paper.

\section{Conclusion}

We have presented a novel method for recovering fundamental structural properties of a texture pattern. The methods applies the extended grey level difference statistics to yield an interaction map that facilitates description of structure in terms of most significant interactions (or spacing vectors). The experimental results demonstrate that the method can be successfully applied to patterns having different degree of regularity and anisotropy, including very weak structures but excluding random isotropic patterns whose interaction maps are not distinct. The latter can be separated and discarded. Due to its high angular resolution and the capability to evaluate the symmetry of a structure, the approach is well suited for directionality and orientation analysis. We plan to apply the proposed approach to image segmentation, detection of objects, including texture defects, and to document image processing. On the other hand, it is planned to proceed with the structural analysis of the interaction maps to obtain the basic geometric parameters of structures.

\section{References}

[1] A. R. Rao and G. L. Lohse, Identifying high level features of texture perception, CVGIP: Graphical Models and Image Processing, 55, 1993, pp.218-233.

[2] Y. Bonneh, D. Reisfeld, and Y. Yeshurun, Texture discrimination by generalized symmetry, in: Proc. 4th ICCV, Berlin, 1993, pp.261-265.

[3] R. M. Haralick, Statistical and structural approaches to texture, Proc. IEEE, 67, 1979, pp.786-804.

[4] F. M. Vilntrotter, R. Nevatia, and K. E. Price, Structural Analysis of Natural Textures, IEEE Trans. on PAMI, 8, 1986, pp.76-89. 
[5] M. Kass and A. Witkin, Analyzing oriented patterns, CVGIP, 37, 1987, pp.362-385.

[6] A. R. Rao and B. G. Schunk, Computing oriented texture fields, CVGIP: Graphical Models and Image Processing, 53, 1991, pp.157-185.

[7] A. R. Rao and R. Jain, Computerized flow fields analysis: Oriented texture fields, IEEE Trans. on PAMI, 14, 1992, pp. 693-709.

[8] S. Denslow et.al., Statistically characterized features for directionality quantitation in patterns and textures, Pattern Recognition, 26, 1993, pp.1193-1205.

[9] M. M. Gorkani and R. W. Picard, Texture orientation for sorting photos "at a glance", Proc. 12th ICPR, I, Jerusalem, 1994, pp.459-464.

[10] H. Greenspan et.al., Rotation invariant texture recognition using a steerable pyramid, Proc. 12th ICPR, II, Jerusalem, 1994, pp.162-167.

[11] F. Liu and R. W. Picard, Periodicity, directionality, and randomness: Wold features for perceptual pattern recognition, Proc. 12th ICPR, II, Jerusalem, 1994, pp.184-189.

[12] D. Chetverikov, Textural anisotropy features for texture analysis, Proc. IEEE Conf. on PRIP, Dallas, 1981, pp.583-588.

[13] D. Chetverikov, Experiments in the rotation-invariant texture discrimination using anisotropy features, Proc. 6th ICPR, Munich, 1982, pp.1071-1073.

[14] I. Hargitai, editor, Symmetry 2: Unifying Human Understanding, Pergamon Press, 1989.

[15] J. Bigün and J. M. H. du Buf, $N$-folded symmetries by complex moments in Gabor space and their application to unsupervised texture segmentation, IEEE Trans. on PAMI, 16, 1994, pp.81-87.

[16] F. Labonté, Y. Shapira, and P. Cohen, A perceptually plausible model for global symmetry detection, Proc. 4th ICCV, Berlin, 1993, pp.258-263.

[17] D. Chetverikov, GLDH based analysis of texture anisotropy and symmetry: an experimental study, Proc. 12th ICPR, I, Jerusalem, 1994, pp.444-448.

[18] S. W. Zucker and D. Terzopoulos, Finding structure in co-occurrence matrices for texture analysis, CGIP, 12, pp.1286-1303.

[19] R. W. Conners and C.A. Harlow, Towards a structural texture analyzer based on statistical methods, CGIP, 12, 1980, pp.224-256.

[20] H.-B. Kim and R.-H. Park, Extracting spatial arrangement of structural textures using projection information, Pattern Recognition, 25, 1992, pp.237-245.

[21] D. Chetverikov, Generating contrast curves for texture regularity analysis, Pattern Recognition Letters, 12, 1991, pp.437-444.

[22] G. L. Gimel'farb, Texture Modeling by Multiple Pairwise Pixel Interaction, submitted to IEEE Trans. on Pattern Anal. Machine Intell.

[23] D. Chetverikov, Pattern Orientation and Texture Symmetry, to appear in Proc. 6th International Conference on Computer Analysis of Images and Patterns (to be published in Springer LNCS series), Prague, 1995.

[24] P.Brodatz, Textures: a photographic album for artists and designers, New York, Dover, 1966.

[25] H.Greenspan et.al., Rotation-Invariant Texture Recognition Using a Steerable Pyramid, Proc. 12th ICPR, II, Jerusalem, 1994, pp.162-167. 